\title{
34 Ethanol - small molecule, strong toxin
}

Alcohols are chemical hydrocarbons where a hydrogen atom is substituted by the functional hydroxyl group (-OH). Ethyl alcohol is the only form that is palatable for humans. It derives from ethane and is thus a 2-carbon compound.

In our world of today, alcoholic drinks are very prevalent. One major reason for their increased consumption is the enormous investment in advertising by the alcohol industry to promote their products. In Germany, they amounted to around $€ 562$ million in 2012. 10.8 million citizens of the Federal Republic of Germany drink alcohol in amounts that pose a risk to their health. According to the Drug Report commissioned by German Federal Government, approximately 1.4 million people between 18 and 65 years of age and 400,000 people over 65 suffer from alcoholism. The risk of a subsequent alcohol addiction is higher the earlier adolescents start drinking alcohol. And this risk is especially high when alcohol consumption starts during puberty, because the brain is in a very critical maturation stage when the decline in cerebral blood flow begins (Satterthwaite et al. 2014).

Alcohol is a cytotoxin that when enjoyed regularly even in small amounts damages nearly all organs in the body. Via acetaldehyde, an intermediate of the biological metabolism of alcohol, it increases the risk for cancer of the oral and pharyngeal cavity, larynx, esophagus, breast, stomach, liver, pancreas and bowel (Allen et al. 2009, Schütze et al. 2011).

In addition to frequent episodes of atrial fibrillation (Kodoma et al. 2011), potential sequelae of excessive alcohol intake include: Elevated blood pressure, myocardial infarction, stroke, inflammation of the liver, pancreas, stomach and bowel, fatty liver, liver cirrhosis, diabetes and nerve cells damage

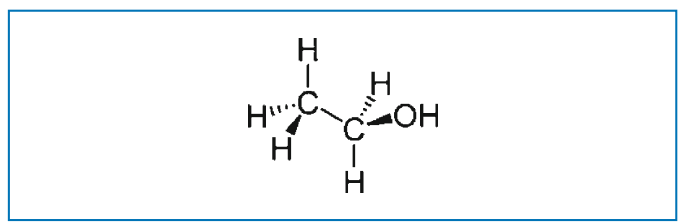

Fig. 34.1 Structural formula of alcohol

accompanied by increased irritability, depression, psychoses and young-onset dementia (Nordström et al. 2013). Life expectancy is markedly limited (Zaridze et al. 2014).

The socioeconomic damage caused by alcohol abuse is tremendous. In the year 2013, the costs in Germany were cited at $€ 27$ billion annually. This stands in contrast with the alcohol tax levied of $€ 3.4$ billion. Every day, approximately 40 alcohol-related fatalities occur in Germany, and 2.5 million worldwide annually according to data from the WHO. Therefore, if at all, alcohol should not be drunk every day: Women should not drink more than $10 \mathrm{~g}$ and men not more than $20 \mathrm{~g}$ of pure alcohol a day. Even moderate alcohol consumption decreases total brain volume, in females more than in males (Paul et al. 2008). The smallest amounts of alcohol are taboo for women during pregnancy - from the beginning to the end.

An intoxicated binge permanently destroys the function of millions of brain cells.

Because alcoholic fermentation occurs in fresh fruit and alcohol is similarly formed by lactic acid fermentation, even healthy produce will contain trace amounts of alcohol, albeit not harmful even to children, according to current knowledge. 